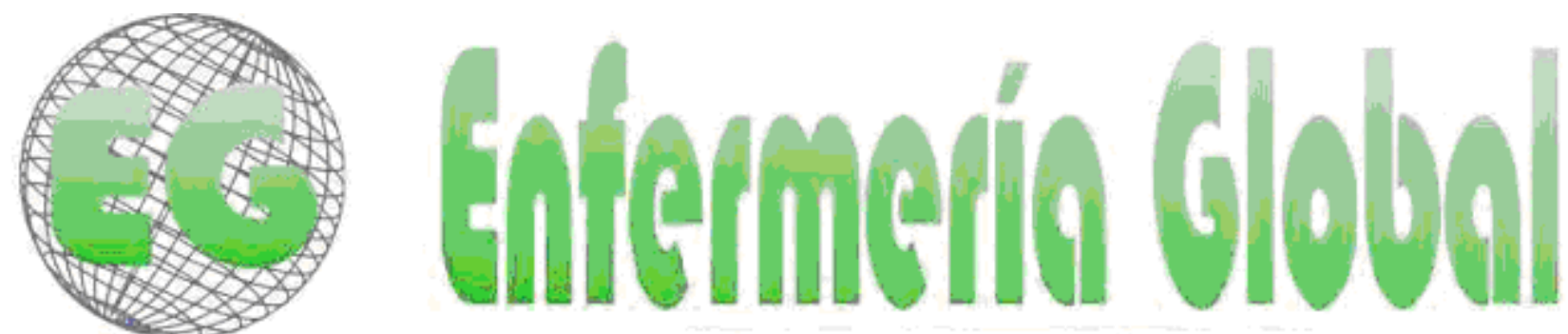

ISSN 1696-6)कS

Prevista electrônica cuatrimestral de Enfermeria

$N^{\circ} 18$

Febrero 2010

www.um.es/egloball

DOCENCIA - FORMACIÓN

\title{
EL SIGNIFICADO DE LA FORMACIÓN PROFESIONAL PARA EL GRADUANDO DE ENFERMERIÁ DE LA UFF FRENTE A SU FUTURO PROFESIONAL
}

O SIGNIFICADO DA FORMAC̃̃O PROFISSIONAL PARA O GRADUANDO DE ENFERMAGEM DA UFF FRENTE AO SEU FUTURO PROFISSIONAL.

*Fonseca da Silva, G., " Marinho Chrizostimo, M., ${ }^{* * *}$ Machado Tinoco Feitosa

Rosas, AM

*Enfermera Graduada. **Enfermera Docente. Mestre en Educación. ***Doctora en Enfermería. Miembros do NUPESENF da EAN da UFRJ. Brasil.

Palabras clave: Educación en Enfermería. Educación Superior. Enfermería

Palavras chave: Educação em Enfermagem. Educação Superior. Enfermagem.

Key words: Education in Nursing, Higher Education, Nursing.

\section{RESUMEN}

El Curso de Graduación de Enfermería de la Universidad Federal Fluminense tiende a propiciar atender el deseo personal y profesional del graduando, en cuanto profesional y en la prestación de la asistencia al cliente de forma humanizada y cualificada. El presente estudio tiene como objeto de estudio el significado del Curso de Graduación para el graduando de enfermería en cuanto a su expectativa y a su futuro profesional. Se trata de una pesquisa cualitativa, con abordaje fenomenológico en que los sujetos fueron 16 discentes del noveno período de la Escola de Enfermagem Aurora de Afonso Costa. Fue posible evidenciar, que la relación entre el docente, el discente y el contenido técnico-científico impartido, desvela fuerte influencia sobre esos discentes en el área de actuación, asociada con exigencia del mercado de trabajo. Ya que el docente, en este contexto, interactúa con el discente en la estructuración del aprendizaje, tendiendo a suplir las perspectivas generadas por estos sujetos, durante su curso académico, mirando a formar un profesional capacitado. Por lo tanto, el objetivo propuesto fue alcanzado, al haber sido posible comprender el significado de la acción intencional del graduando del Curso de Enfermería, con relación a la expectativa de su futuro profesional.

\section{RESUMO}

O Curso de Graduação de Enfermagem da Universidade Federal Fluminense tende propiciar atender o anseio pessoal e profissional do graduando, enquanto profissional e na prestação da assistência ao cliente de forma humanizada e qualificada. O presente estudo tem como objeto de estudo o 
significado do Curso de Graduação para o graduando de enfermagem quanto à sua expectativa e o seu futuro profissional. Trata-se de uma pesquisa qualitativa, com abordagem fenomenológica em que, os sujeitos foram 16 discentes do nono período da Escola de Enfermagem Aurora de Afonso Costa. Contudo, foi possível evidenciar, que a relação entre o docente, o discente e o conteúdo técninco-cientifico ministrado, desvela forte influência sobre esses discentes na área de atuação, associada com exigência do mercado de trabalho. Já que o docente, neste contexto, interage com o discente na estruturalização do aprendizado, tendendo suprir as perspectivas geradas, por estes sujeitos, durante seu percurso acadêmico visando formar um profissional capacitado. Portanto, o objetivo proposto foi alcançado, visto ter sido possível compreender o significado da ação intencional do graduando do Curso de Enfermagem, com relação à expectativa do seu futuro profissional.

\section{INTRODUCCIÓN}

La situación problema emerge de la precisión de comprender la perspectiva del Graduando de Enfermería de la Universidade Federal Fluminense, frente a la formación y su futuro profesional. Esa busca se dio a partir de las observaciones identificadas por los graduandos durante el trayecto académico. Estas aspiraciones aumentaron ante la proximidad del término del curso de Enfermería, cuando al asumir nuevo ambiente y nueva posición, afloran sentimientos reales o imaginarios influyentes en el actuar del hombre en sí. Esto es una acción inherente del ser humano mediante un proceso de cambio, en el cual se es permisivo en todas las fases del su desarrollo evolutivo, como se ratifica más adelante.

Mudanza es el proceso de transformación de un ser, de un ambiente o de una situación en un nuevo ser, nuevo ambiente o nueva situación, en razón de alteraciones cualitativas y/o cuantitativas, que mira a la mejoría, al perfeccionamiento y al desarrollo. $Y$ un proceso porque se trata de la acción del movimiento de transformación en el tiempo y en el espacio, para dar una nueva forma, facción, carácter o dirección a alguna cosa, tornandola diferente de la que era ${ }^{1}$.

El modo como cada uno se enfrenta con las situaciones vivenciadas es el resultante de la influencia que recibió a lo largo de su vida, a través de su proceso educativo en la construcción de los valores. Los valores en la educación forman la persona humana en su unidad, en la que los aspectos cognitivos, afectivos, prácticos y espirituales no están disociados o funcionan separadamente. Mas, los valores están ligados a la experiencia y al sentimiento como estado afectivo, mientras que emoción es toda manifestación compleja y organizada de la vida afectiva. Ellos se encuentran en un envolvimiento en el que está comprometida la persona humana en su totalidad ${ }^{2-3}$.

Por consecuencia, este es uno de los factores también actuante en el crecimiento del individuo y en la determinación profesional, se puede afirmar que una preparación educacional edifica el sujeto, en el aspecto escolar, lo que causa seguridad para su aplicación práctica como también para todo contexto de vivencia que se infiera.

Así, el objeto de estudio es el significado del Curso de Graduación para el graduando de enfermería en cuanto a su expectativa sobre su futuro profesional. Por lo tanto, este estudio tiene como objetivo: Comprender el significado de la acción intencional, del graduando del Curso de Enfermería, con relación a la expectativa del su futuro profesional.

Tiene como cuestión orientadora el siguiente cuestionamiento: ¿Será que el estudiante identifica el Curso de Graduación en Enfermería como una posibilidad de alcance del futuro profesional? 
La relevancia del estudio está en la reflexión y discusión con el docente y discente sobre el área de actuación asociada con la visión del mercado de trabajo, lo que genera influencia mutua entre la formación del estudiante y el contexto del trabajo. El educador interactúa en su estructura, lo que viene a contribuir a la reflexión y discusión entre los graduandos y docentes sobre este proceso y el desarrollo académico del discente.

De tal modo, el alumno durante su graduación ansía el "saber" en beneficio de su carrera profesional, así se presenta el problema de este estudio que consiste en la acción intencional del alumno en cuanto a su saber técnico-científico e inclusión en el área de actuación. Sin embargo, este conocimiento podrá no atender con eficacia sus necesidades para ingresar en el campo de la ocupación pretensa.

En este contexto, el estudiante para alcanzar su objetivo, primariamente precisa estar comprometido consigo y con el mundo en que vive, ser capaz de actuar, ausentarse del ámbito teórico y reflexionar sobre la realidad vivida y el trabajo a ser desarrollado por él.

\section{MATERIAL Y MÉTODO}

\section{La opción por el referencial fenomenológico}

El primer contacto con la teoría fenomenológica se dio en el tercero período, con la disciplina obligatoria curricular del Curso de Graduación de Enfermería, en Metodología de la Pesquisa. En este momento se presentaron las tres principales corrientes Filosóficas Positivismo, Fenomenología y Marxismo.

Iniciada la construcción del presente estudio, en forma de anteproyecto, ya en el séptimo período, en 2007, surgió la necesidad de basarlo en una de esas ciencias, de entre las cuales remití la fenomenología. Siendo esta un método investigativo del fenómeno ocurrido, centrado en exposición del ser humano, lo que describe la exhortación de presupuestos y explicaciones. Capta lo íntimo de la acción, la esencia emitida de inmediato, al luchar con la subjetividad de los sujetos abordados que son actores de su propia existencia.

La fenomenología procura abordar el fenómeno, aquello que se manifiesta de modo que no lo parcializa o explica a partir de conceptos previos, de creencias o afirmaciones sobre lo mismo, en fin, de un referencial teórico. Mas ella tiene la intención de abordarlo directamente, interrogándolo, intentando describirlo y procurando captar la esencia ${ }^{3}$

Es dentro de las ciencias sociales donde el fundamento metodológico de la fenomenología es tratado y discutido por el filósofo alemán Edmund Husserl al final del siglo XIX e inicio del siglo XX, con el propósito de hacer de esta una ciencia. Este, a través de las influencias de Weber, argumenta que "los actos sociales envuelven una prioridad que no está presente en los otros sectores del universo, abarcados por las ciencias naturales: el significado". La fenomenología es la doctrina universal de las esencias, en que se integra la ciencia de la esencia del conocimiento ${ }^{4-6}$.

La fenomenología defiende la idea de que la realidad social se fundamenta en los significados y solo se pueden desvelar a partir del momento en que se integra en el lenguaje expresivo de la interacción social, o sea, la relación humana. Comunicación competente es un proceso interpersonal que debe alcanzar el objetivo de los comunicadores; presupone conocimientos básicos de comunicación; que los implicados tengan consciencia de lo verbal y no verbal en las interacciones; exige claridad y objetividad; promueve el autoconocimiento 
y posibilita una vida más auténtica ${ }^{7}$.

\section{Aspectos de la etapa de campo}

La pesquisa fue realizada en la Escola de Enfermagem Aurora de Afonso Costa, de la Universidade Federal Fluminense, localizada en el municipio de Niterói. El período de realización de las entrevistas se dio en el $1^{\circ}$ semestre de 2008 . La pesquisa está constituida de abordaje cualitativa basada en teoría fenomenológica ya anteriormente descrita.

La Escola de Enfermagem Aurora de Afonso Costa, actualmente, está compuesta de una biblioteca; laboratorio de informática, (recientemente inaugurado); salas de aulas de diferentes dimensiones; sala de acogida para los alumnos, espacio académico de enfermería Edézio A. de Souza y Vivian R. Gomes; Directorio académico con instalaciones propias para las refecciones y demás dependencias para el funcionamiento adecuado al curso de graduación y laboratorio para el perfeccionamiento técnico de las disciplinas prácticas. Posee equipamientos de recursos audiovisuaesl para administrar aulas teóricas.

En el sector técnico-administrativo, el equipo está formado por personal administrativo, funcionarios de limpieza y equipo de seguridad 24 horas, divididos en turnos. Estructuralmente, posee seis plantas para ejecución de aulas y servicios. Cada planta se distribuye en un departamento correspondiente y sus coordinadores para la graduación y pos-graduación. En el bajo se encuentra un auditorio que se denomina Prof. a Dr. a Rosalda Paim donde se realizan numerosos eventos.

La Escola de Enfermagem da Universidade Federal Fluminense-UFF adopta el sistema Nightingel desde su génesis, así como la mayoría de las demás escuelas de enfermería en Brasil. En el Parecer del Conselho Federal de Educação - CFE de no. 163/72 el curriculum mínimo expreso fue realizado en tres años lectivos, después de dos años fue ampliado para un año más por el Conselho de Ensino de Pesquisa da Universidade Federal Fluminense UFF. La enfermería de la Universidade Federal Fluminense tiene como característica general que forma profesionales preparados para ingresar tanto en la red hospitalaria cuanto en la red básica de salud. En el capítulo siguiente serán pormenorizados la génesis y el funcionamiento de la Escola de Enfermagem Aurora de Afonso Costa - EEAAC.

La referida pesquisa obtuvo autorización del Comité de Ética de la Faculdade de Medicina/ Hospital Universitário Antônio Pedro de la Universidade Federal Fluminense. Esta fue grabada en aparato digital de captura de audio y digitada enseguida íntegramente, para no perder ningún dato de la pesquisa. Los sujetos de este estudio son los alumnos del Curso de Graduación en Enfermería del noveno período. La elección de este grupo está en íntima relación con el término del Curso, con las perspectivas ante su formación y su ingreso profesional.

\section{LA CONSTRUCCIÓN DEL CONOCIMIENTO TEÓRICO}

\section{La contextualización de la enseñanza superior y la enfermería en Brasil}

En el abordaje de la contextualización de la enseñanza superior en Brasil es fundamental mirar para la educación brasileña y su influencia en la formación del hombre pues la educación es la base de la formación del desarrollo humano en la vertiente tanto intelectual como moral, o sea, comprende factores hereditarios y adaptaciones biológicas dependientes directamente de la evolución del sistema nervoso y mecanismos psíquicos elementales y de las interacciones sociales, que sumados construyen progresivamente a lo largo de la vida su 
carácter de comportamiento y mental ${ }^{8}$.

En ese contexto todo individuo tiene derecho a la educación. Luego, hablar de ese derecho es admitir el papel imprescindible de los factores sociales en la formación del ser humano. Como también, los derechos y deberes del ciudadano están incluidos en la Constitución de Brasil, llamada Carta Magna, sentando así la base de la organización brasileña en todos los aspectos. Esta constitución promulgada en 1988 establece en el art. 205 que: La educación, derecho de todos y deber del Estado y de la familia, será promovida e incentivada con la colaboración de la sociedad, lo que apunta al pleno desarrollo de la persona, a su prepararación para la enseñanza de la ciudadanía y su cualificación para el trabajo ${ }^{8-9}$.

\section{La enseñanza superior}

En la actualidad, se discute acerca de la cantidad y calidad de cursos de niveles superiores en Brasil, donde hasta finales del siglo XIX existían sólo 24 establecimientos de enseñanza superior, con cerca de 10.000 estudiantes $^{10}$.

En 1808, se inicia la enseñanza superior en Brasil con la llegada de la familia real portuguesa al país. Sin embargo, la iniciativa privada y la "expansión" de la enseñanza superior solamente acontecieron mucho tiempo después con la Constitución de la República de 1891 que descentralizó la oferta de enseñanza superior, lo que permitió que los gobiernos estaduales y la iniciativa privada creasen su propio establecimiento ${ }^{11}$.

En la década de los 30, las primeras estadísticas sobre la educación contaban con $64,4 \%$ de instituciones en la iniciativa privada, siendo relativamente constante durante treinta años seguidos. La demanda no absorbida por el estado abrió espacio a la iniciativa privada.

De este período hasta la actualidad, la enseñanza superior en Brasil contó con significativos cambios, tanto en el funcionamiento como en su configuración, tales como titulación de los docentes, institucionalización de la pesquisa y de la producción intelectual, calidad de la formación ofrecida, diversidad de oferta de cursos, entre otros ${ }^{11}$.

En la década de 1990, según un estudio, la proporción de estudiantes oriundos de familias con renta por encima de 10 salarios mínimos sobrepasaba el $60 \%$, tanto en el sector público como en el privado. Se observó que el acceso a la enseñanza superior era mayor entre la clase social más elevada lo que discierne la "creencia" de que los menos favorecidos frecuentan la enseñanza privada. Tal hecho se da a la inversa de lo que se imagina, no por falta de plazas. Lo que ocurre es una deficiencia en la enseñanza fundamental además de problemas sociales.

El hecho es que, expresivas y significativas mudanzas acompañan los días actuales y de la misma forma - independiente de la clase social - la enseñanza superior. No obstante el crecimiento y demanda por cursos en nivel superior, la diversidad se hace una constante.

En la enseñanza superior brasileña las discusiones fueron intensas con: el Ministério da Educação en la formulación de la Ley de Directrices Básicas de la Educación (LDB) Nacional; la Associação Brasileira de Enfermagem en la promoción de los Seminarios Nacionales de Directrices para la Educación en Enfermería en Brasil; las Escuelas de Enfermería con las reformulaciones de los Proyectos Políticos Pedagógicos (PPP), en las directrices curriculares y efectivamente en los currículos.

El Proyecto Político Pedagógico (PPP) de la Universidade Federal Fluminense, parte de una 
visión de totalidad sobre la formación del profesional. El gran desafío será superar la visión fragmentada-fragmentador y de compartimento de los binomios salud-dolencia, alumnoprofesor, integración vertical-horizontal, relación teoría-práctica, procedente de los modelos que predominan históricamente en la formación del enfermero ${ }^{12}$.

\section{La enfermería en Brasil}

Al hablar de enfermería técnico - científico, no se puede dejar de mencionar a la pionera de este estudio epistemológico, Florence Nightingale, un mito de la génesis de la categoría, precursora de la enfermería profesional y marco de la Enfermería moderna, para el entrenamiento práctico y la formación moral del enfermero ${ }^{13}$.

Florence nació el 20 de mayo de 1820, en Florencia, Italia. Perteneciente a la alta casta de la sociedad inglesa fue educada por preceptores para asumir funciones que la sociedad determinaba a las mujeres - el casamiento. Al desafiar las interpretaciones sociales y culturales de su época, se tornó icono de mujer como fuerza de trabajo y ganancia en el espacio público. Renuncio a someterse a la vida de esposa para transitar un camino arduo para la realización de su sueño de ser nurse. Con la Guerra de Crimea, Florence ve una oportunidad de proseguir con sus ideales y concretizarlos e instituyó tras la guerra una escuela de enfermería en el Hospital Saint Thomas que desde entonces pasó a ser un referencial para las demás escuelas ${ }^{14}$.

La enfermería emerge ahora no como una práctica empírica, mas como una ocupación asalariada que viene a atender la necesidad de mano de obra en los hospitales, constituyendose como una práctica social institucionalizada y específica. Para Florence, el foco principal era el doliente y no la enfermedad, en la cual las acciones de salud auxilian la acción de la fuerza de la naturaleza ante la creación de condiciones que favorecieran el proceso de cura $^{15}$.

De esa forma Florence incorpora los siguientes conceptos: el ser humano, el medio ambiente en que este se encuentra, la salud y la enfermería. Estos convergen en un mismo objetivo y en lo que hoy se identifica como bases humanísticas de la Enfermería, en la filosófica teoría de la Enfermería holística, que preconiza el cuidar del ser en un todo.

En Brasil la enfermería se consagró bajo la instauración, en la década de 20, del Sistema Nightingale por Carlos Chagas, director del Departamento Nacional Saúde Pública-DNSP. En este período el Brasil pasaba por una recesión económica tras la guerra, donde la población estaba sometida a las pésimas condiciones de vida y las epidemias proliferaban en la ciudad ${ }^{16}$.

Así, hubo necesidad de alistar enfermeras habilitadas para actuar en la reforma sanitaria, apoyadas por la Fundação Rockefeller, que envió una enfermera Ethel Parson implantando la Misión Técnica de Cooperación de Desarrollo de Enfermería en Brasil, creando escuelas para habilitar a enfermeras brasileñas para el trabajo adjunto a los problemas de salud pública y estar aptas para substituir a las enfermeras extranjeras de la Misión ${ }^{17}$. Esa Escola de Enfermeiras do Departamento Nacional Saúde Pública-DNSP es hoy la Escola de Enfermagem Anna Nery. Aunque la consagración de la Enfermería fue a partir de 1920, cabe retomar un poco la historia y hacer un breve trazado de su constitución en Brasil. 


\section{La génesis de la Escola de Enfermagem Aurora de Afonso Costa - EEAAC}

La Escola de Enfermagem surgió en abril de 1944, como Universidade Federal Estadual de Rio de Janeiro ${ }^{18}$. En esa época Brasil se encontraba bajo la dictadura de Vargas, sin cuestionar el régimen vigente cabe resaltar que este benefició a algunos Estados para la ascensión técnica y administrativa, entre estos el Estado de Rio de Janeiro. La idea de fundar una escuela de enfermería surgió debido a un vasto programa sanitario que fue creado para atender el Estado89 o de Rio, bajo la intervención de Amaral Peixoto. Siendo un proyecto de grandes dimensiones, sería preciso para atender su objetivo la necesidad de profesionales de alto padrón para atender los servicios asistenciales y también en los centros de salud, creados en ese mismo período.

En 1943, se pensó la realización de esa obra en Campos, al considerar que esta región era privilegiada por su localización, un cuerpo docente de calidad, red hospitalaria en ascensión, por tener un amplio campo de prácticas, entre otros factores, pero fue la capital del Estado Niterói, la que alcanzó éxito para ser elegida como sede de la nueva universidad.

Así, se considera que en homenaje al presidente Getúlio Vargas, en 19 de abril de 1944, en la Assembléia legislativa do Estado do Rio de Janeiro, estaban reunidos los miembros de la Comissão Administrativa, liderada por el comandante Ernani do Amaral Peixoto y otras autoridades, los cuales asignaran el Decreto - ley no. 1130, lo que da al territorio de Niterói el aval para la génesis de la Escola de Enfermagem do Estado do Rio ${ }^{18}$.

La clase pionera tenía en gran mayoría, discentes de la región norte del país. El programa didáctico impartido se basaba en las entidades oficiales precedentes. En conmemoración del primer año de funcionamiento, se realizó la primera ceremonia de recepción de Toca Simbólica, en homenaje a la Dama de la Lampada.

\section{EL ENFERMERO Y LOS FACTORES QUE INTERFIEREN EN LA ENSEÑANZA- APRENDIZAJE}

\section{El ser enfermero}

El enfermero busca respetar la vida y la dignidad de la persona humana, según el Código de Ética de los profesionales de enfermería. El profesional de la enfermería respeta la vida, la dignidad y los derechos de la persona humana, en todo su ciclo vital, la discriminación de cualquier naturaleza, asegura al cliente una asistencia de enfermería libre de daños derivados de impericia, negligencia o imprudencia, cumple y hace cumplir los preceptos éticos y legales de la profesión, ejerciendo la enfermería con justicia, competencia, responsabilidad y honestidad ${ }^{19-20}$.

La ley del ejercicio profesional desvela que la enfermería es libre en todo territorio nacional, lo que admite que sus actividades sean realizadas por individuos legalmente habilitados de acuerdo con lo dispuesto en la jurisdicción e inscritas en el Conselho Regional de Enfermagem. La ley del ejercicio profesional dispone sobre el ejercicio de la profesión de enfermería, dice que el Enfermero es aquel que obtiene titulación, diplomado Enfermero y/o Enfermero Obstetra que le es conferida por la institución en los términos coherentes a la ley 21 


\section{Factores influyentes en el proceso enseñanza-aprendizaje}

Se puede decir que hoy la educación general y la educación profesional comenzaron a ser aceptadas como interrelacionadas, principalmente, por dos procesos: la globalización, que trae una multiplicidad de cambios surgidos a partir de 1970, que instituyó nuevas relaciones en ámbito internacional en los planos económico, social, cultural, político y tecnológico y la emergencia de un sistema de producción sustentado en la automación flexible. Actualmente, dentro del contexto bio psicosocial y económico-cultural se considera la complejidad del hombre y el medio en que vive, lo que viabiliza recursos para la formación del profesional competentes para lidiar con los desafíos del siglo XXI.

En el campo de la enfermería, esta categoría presenta los sentimientos vivenciados por los estudiantes durante el curso, los cuales manifiestan de forma diversificada que se modifica y se alterna desde la euforia por el paso a la universidad, realización, alegría, curiosidad, interés en crecer, orgullo por hacer enfermería, hasta sentimientos de angustia, tristeza, miedo, terror, fracaso, soledad, sensación de desamparo, inferioridad, incapacidad, decepción, voluntad de desistir, rabia, revuelta, inseguridad, pena, sufrimiento y dolor. La convicción de que el profesional de salud, al trabajar con personas y luchar con sus emociones, sentimientos, no consigue colocar su propia persona al margen de ese proceso, de modo que el contacto establecido por el profesional en el momento del cuidado, puede suscitar la alteración de sus propios sentimientos.

Los estudiantes dejan explícitos sentimientos, como falta de seguridad y miedo, cuando perciben que tendrán que actuar junto al paciente con la actitud de un profesional. Esos sentimientos se justifican por la dificultad en la interacción y en la comprensión de la comunicación paciente-alumno, cuya preocupación mayor es la sensación de prejuicio que puede ser causado al paciente, por sus inhabilidades y conocimientos aún limitados ${ }^{22}$.

\section{LOS RESULTADOS DEL ESTUDIO}

\section{La vivencia con abordaje teórico-metodológica}

Al considerar la vivencia con el abordaje teórico-metodológico cabe resaltar cómo fue elaborada la pesquisa. De esta manera, se remite al estudio cualitativo con abordaje fenomenológico de la sociología comprensiva de Alfred Schütz ${ }^{23}$, que ante el objeto de estudio que es el significado del curso de graduación para el graduando de enfermería en cuanto a su expectativa y a su futuro profesional, objetiva comprender el significado de la acción intencional del graduando del curso de enfermería, con relación a la expectativa del su futuro profesional.

Así, se desvelan los sujetos de la pesquisa que fueron los graduandos de enfermería del último período de la Universidade Federal Fluminense. El escenario fue la Escola de Enfermagem Aurora de Afonso Costa, donde fueron realizadas algunas entrevistas y otras se dieron en el Hospital Universitário Antônio Pedro, con horario y local acordado. La diferenciación de local se dio debido a la división de la clase en subgrupos, teniendo en cuenta que las disciplinas poseen enmiendas apropiadas al contenido programático y necesitan de campo de prácticaas diferentes para cada subgrupo.

El mecanismo de identificación de los estudiantes para mantener su anonimato fue el nombramiento que cada sujeto emitió para resumir el curso de graduación en una única palabra, esta transcrita en el término de los relatos. De entre los 16 sujetos entrevistados se apunta el perfil de los discentes. La mayoría eran solteros, sin hijos, moradores y 
dependientes financieramente de padres /familiares, naturales del estado del Rio de Janeiro.

Se observa que ínfima parte posee renta propia y que son actuantes en el mercado de trabajo. Y aún, los participantes de la pesquisa son del sexo femenino con faja de edad variante entre 21 a 36 años, que se encuentran dentro de la población económicamente activa. Según datos del IBGE de noviembre de 2006, la distribución de la población económicamente activa por faja de edad apuntó que: 0,3\% estaban en la faja de 10 a 14 años de edad; 2,4\%, de 15 a 17 años; 18,1\%, de 18 a 24 años; $61,4 \%$, de 25 a 49 años y $17,8 \%$, de 50 años o más.

Dentro del grupo de los entrevistados, se reveló la predominancia del género femenino, ya comprendido por la historia natural de las Escuelas de Enfermería. Identificó también una amplia participación de esos discentes en actividades complementarias ofrecidas y reglamentadas por el Colegiado de Curso. De acuerdo con el Proyecto Pedagógico del Curso son actividades que posibilitan la permanente y contextualizada actualización profesional a través de la práctica de estudios independientes, transversales, opcionales, pudiendo incluir actividades de Enseño, Pesquisa, Extensión y Académico-Administrativas. Vale resaltar que fueron cumplidas las exigencias de la ley $196 / 96^{24}$ del que dispone sobre los preceptos éticos para pesquisa con seres humanos.

\section{DISCUSIÓN}

\section{El desvelar del análisis comprensivo: el graduando frente a la formación.}

El curso de graduación conduce a la instrumentalización de los alumnos para la formación de futuros profesionales en el área de salud. Y que el docente debe estar atento al proceso de enseñanza-aprendizaje que es su papel en el contexto de la educación de los discentes, en la práctica profesional y en el relacionamiento interpersonal. Así, la forma coherente de transmitir al alumno todo el esbozo teórico y práctico desvela el curso de graduación de enfermería, para capacitarlo en el ámbito de la salud.

Al analizar las hablas, se percibe que solamente el diálogo implica en un pensar crítico, y es capaz también de generarlo, sin este no hay comunicación ni hay verdadera educación ${ }^{25}$. Es pertinente también considerar el nombre que cada sujeto emitió para resumir el curso de graduación en una única palabra, transcrita al término de los relatos, la cual fue utilizada como mecanismo de identificación de los estudiantes para mantener su anonimato. Son en ellas donde están contenidos de forma sucinta lo que el curso de graduación significó para cada individuo, lo vivido durante su formación.

De entre diversas cuestiones que emergieron de las hablas de los sujetos, fue retratada la divergencia de sus expectativas en cuanto futuros profesionales de salud, al relacionar su formación general, la formación profesional y las experiencias de trabajo.

El curso podría haber sido mejor [...] y la actuación incluso, del enfermero para ser enfermero eso yo tuve que buscar mucho por cuenta propia [...] Razonable

Es, no atiende a las... es ... las mías, como mínimo mi expectativas... es...en cuanto al mercado de trabajo[...]algunas disciplinas en la escuela de enfermería como actividad interdisciplinar es.., Salud, Sociedad y Enfermería esas disciplinas ahí son totalmente dispensables porque el profesor fingía dar aula y nosotros fingíamos que aprendíamos [...] Entonces, yo particularmente llamaba disciplinas de aumentar coeficiente de rendimiento (CR) y solo. Frustración 
Entonces mi expectativa cuando yo entré era... salir un... formar un profesional capacitado, entrenado, entendió [...] que así, que tuviese, que me diese un soporte para que la gente saliese como mínimo con un poquito más de... de confianza, de dominio de la profesión [...] Despreparo

Siendo la primera categoría: formar el graduando de enfermería con articulación entre la formación general, profesional y las experiencias de trabajo para atender las necesidades de la sociedad y del profesional Enfermero.

Nosotros no tenemos una base como mínimo dentro de la facultad para eso nosotros tenemos que buscar fuera a través de cursos o del propio estudio individual.[...] Frustración

El curso de graduación no queda debiendo en nada, ah... a la mi vida profesional porque... yo encuentro que él fue completo [...] Confianza

Con relación a la expectativa profesional es... Mira, yo creo que preparar,... No prepara totalmente, yo creo que nosotros es... nosotros tenemos que ir a buscar a otros lugares. Hay personas que pueden estar teniendo más experiencia [...] Perfeccionamiento

La segunda categoría: posibilitar al graduando de Enfermería a superar la fragmentación del proceso de la enseñanza teórica y de la práctica a través de la educación continuada del profesional Enfermero.

Tiene mucha parte... mucha parte científica, parte ... de metodología, ahora práctica no tiene, lo que es de mucha importancia para nosotros también[...] Miedo (b)

Hasta usted decir que el trabajo técnico que el enfermero tiene que saber y la facultad no prepara para ello, ello prepara a usted más para... para liderar, para gerenciar, para pesquisa, para el curso master, de repente, un doctorado, si usted continua sus estudios [...] Inseguridad

Entré en la enfermería por casualidad, más en relación al mercado de trabajo yo me vengo adaptando bien, la [...] a facultad y ya estoy empleada [...] Suceso

El curso de graduación conduce a la capacitación de los alumnos para la formación de futuros profesionales en el área de la salud. El docente debe estar atento al proceso del enseñanza-aprendizaje que es su propio papel en el contexto de la educación de los discentes, en la práctica profesional y en la relación interpersonal.

\section{CONCLUSIÓN}

Desde la génesis de la Escuela Profesional de Enfermeros y Enfermeras en Brasil, a partir de 1890, se discute la forma de cualificar a los profesionales para enfermería a fin de suplir debidamente sus objetivos. Mediante dificultades presentadas, consecuentes crisis económicas y políticas, surgió a lo largo de ese período, la necesidad de reorganizar estructuralmente la enseñanza, lo que vino a suscitar, de una forma amplia, el crecimiento intelectual y una consecuente busca de la especialización de la fuerza de trabajo. Y hasta hoy tales similitudes siguen aún vigentes en nuestra realidad.

En ese contexto comprender el significado de la acción intencional, del graduando del Curso de Enfermería, con relación a la expectativa de su futuro profesional, se dio a través de dos categorías, formar al graduando de Enfermería con articulación entre la formación general, 
profesional y las experiencias de trabajo para atender a las necesidades de la sociedad y del profesional enfermero, y posibilitar al graduando de Enfermería para superar la fragmentación del proceso de la enseñanzao teórica y práctica a través de la educación continuada del profesional Enfermero.

Para posibilitar el estímulo y motivación a los discentes que atienden a través de la formación su cualificación, el educador debe percibirse no meramente como dominador del saber, que deposita en los educandos siendo estos pausibles de un falso saber. Este método no promueve la esencia de la educación libertadora, que para tal necesita del diálogo. Así sólo el diálogo provoca el pensar crítico, sin el diálogo no hay comunicación y sin el pensamiento crítico no existe la verdadera educación.

Ciertamente, ese trabajo vino a contribuir a que el sistema capacitador responsable de la preparación profesional sea analizado de forma coherente y significativa.

\section{REFERENCIAS}

1- Xavier OS. A Educação no Contexto das Mudanças. Rev Bras Estudos Pedagógicos 1997;78: 188-190.

2- Cuvillier A. Pequeno vocabulário da língua filosófica. São Paulo: Nacional; 1969.

3- Capalbo C. Metodologia das Ciências Sociais: A fenomenologia de Alfred Schütz.

Rio de Janeiro: Antares Universitária; 1979.

4- Rosas AMF. A Consulta de Enfermagem na Perspectiva dos Enfermeiros na Unidade de Saúde. Rio de Janeiro; 1998. Mestrado [ Dissertação em Enfermagem] - EEAN/UFRJ.

5. Husserl E. Investigações Lógicas. Madrid, Rev. de Occidente 1976.

6- Weber M. Metodologia das Ciências Sociais. Campinas: Unicamp; 1992.

7- Braga EM, Silva MJP. Comunicação competente: visão de enfermeiros especialistas em comunicação. Acta paul. enferm 2007; 20 (4): 410-414.

8- Piaget J. Para onde vai a educação? 8 ed. Rio de Janeiro: Livraria José Olympio, 1984.

9- Brasil. Assembléia Nacional Constituinte da República Federativa do Brasil. Portaria n.ำ 205. Diário Oficial [da República Federativa do Brasil]. Brasília, 1988; Seção 1.

10- Rodrigues JH. História da história do Brasil. Companhia Editora Nacional/MEC; Vol.21, 1979.

11- Silva DA. O ensino superior no Brasil:do séc. XIX aos dias atuais. 24-03-2008. Artigonal [ on line] 2008 [ capturado em Dez. 2008 ]. Disponível em: www.artigonal.com/ensinosuperior-artigos/o-ensino-superior-no-brasil-do-sec-xix-aos-dias-atuais-368028.html.

12- Pró-Reitoria de Assunto Acadêmico - Coordenadoria de Apoio Ao Ensino de Graduação. Niterói; 2005. [Projeto Político Pedagógico da Escola de Enfermagem da Universidade Federal Fluminense - Escola de Enfermagem Aurora de Afonso Costa / UFF ]

13- Daher DV. Por Detrás da Chama Da Lâmpada:A identidade social do enfermeiro. Rio de Janeiro: EDUFF, 2000.

14- Sobral VRS. A Purgação do Desejo: memórias de enfermagem. Rio de Janeiro;1994.Doutorado [tese em Doutorado em Enfermagem] - EEAN/UFRJ.

15- Geovanini T, Moreira A, Dornelles S, Machado WCA. História da Enfermagem: Versões e Interpretações. Rio de Janeiro: Editora Revinter, 1995.

16- Gentil DMI, Chrizostimo MM, Simeão MSS, Rosas AMTF, Oliveira LV. Relación histórica de la consulta de enfermería con la vivencia profesional. Enf. Global 2009;

17- Instituto Oswaldo Cruz. Casa de Oswaldo Cruz. Fiocruz. Dicionário Histórico-Biográfico das Ciências da Saúde no Brasil [on line]. Rio de Janeiro, Brasil; 2007.

[capturado em 26 set. 2007] Disponível em http://www.dichistoriasaude.coc.fiocruz.br

18- Fernandes AFC, Rodrigues MSP, Cavalcanti PP. O comportamento da mulher 
mastectomizada frente às atividades grupais. Rev Bras Enferm 2004; 57(1): 31-4.

19- Passos G S, Chrizostimo M M, Rosas AMTF. Consulta de enfermagem: um olhar atento à mulher acometida pelo câncer de mama. Niterói; 2007. Trabalho de conclusão de curso [ Monografia em enfermagem] - Escola de Enfermagem Aurora de Afonso Costa da Universidade Federal Fluminense.

20- COFEN - CONSELHO FEDERAL DE ENFERMAGEM. COREN/GO. Código de Ética dos profissionais de Enfermagem. Brasília (DF) 2000.

21- ASSOCIAÇÃO BRASILEIRA DE ENFERMAGEM. A 'nova' Lei do Exercício profissional da enfermagem. Brasília: ABEn, 1987. (caderno de Legislação/documentos).

22- Garro IMB, Camillo SO, Nóbrega MSSP. Depressão em Graduandos de Enfermagem. Acta Paul Enfermagem, 2006; 19 (2): 162-7.

23- Alfred S. Fenomenologia e relações sociais textos escolhidos de Alfred Schütz. Rio de Janeiro: Editora Zahar; 1979.

24- BRASIL, Ministério da Saúde. Conselho Nacional de Saúde. Resolução CNS 196/96. Brasília, 1996.

25- Freire P. Pedagogia do Oprimido. 30 ed. Rio de Janeiro: Paz e Terra; 2001. 